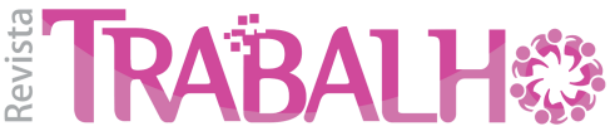 \\ (En)Cena
}

\section{CONTRIBUTIONS AND LIMITATIONS OF THREE INTERPRETATIVE PERSPECTIVES ON THE DIFFICULTIES OF TEACHING AND LEARNING MATHEMATICS AT THE ELEMENTARY LEVEL}

Apports et limites de trois perspectives interprétatives des difficultés d'enseignement et d'apprentissage en mathématiques chez les élèves du primaire

Contribuciones y límites de tres perspectivas interpretativas de las dificultades de enseñanza y aprendizaje en matemáticas en los alumnos de primaria

Aportes e limites de três perspectivas interpretativas das dificuldades de ensino e aprendizagem da matemática dos alunos do primário

Thomas Rajotte ${ }^{1}$

Université du Québec à Rimouski

Profess eur à l'Université du Québec à Rimouski, campus de Lévis (UQAR-Lévis). Ses intérêts de recherche porte sur la résolution de problèmes mathématiques, les difficultés d'apprentissage en mathématiques ainsi que sur la pédagogie par le jeu.

\begin{abstract}
Abs tract
The purpose of this paper is to shed some light on the contributions and limitations of three different perspectives on teaching difficulties and learning disabilities in mathematics at the elementary level. More particularly, this article follows on various studies seeking to document the fundamental source of these problems. A review of the mostly French-language academic literature produced over the past 30 years uncovers three broad points of view regarding students with learning disabilities in mathematics. The first, which is rooted in the cognitive sciences, attributes these learning disabilities to students' inherent characteristics. The second considers that these difficulties stem from the interaction between the student and the teaching system. The third perspective relies on the foundations of sociology to interpret the learning difficulties experienced by students in mathematics. The purpose of this paper is to describe these three perspectives and to paint a picture of the different tools used by their proponents to overcome problems related to teaching and learning mathematics. The article also offers up a brief reflection on the opportunity of bringing these different points of view together.
\end{abstract}

Keywords: Learning disabilities, didactics of mathematics, sociology, anthropo-didactic approach, cognitive sciences.

\section{Résumé}

Ce texte vise à mettre en lumière les apports et les limites des différentes perspectives qui relèvent de l'interprétation des difficultés d'enseignement et d'apprentissage en mathématiques des élèves du primaire. Cet article se situe plus précisément dans la foulée des recherches permettant de s'interroger sur la source fondamentale de ces difficultés. Un regard sur les écrits scientifiques, relevant principalement du corpus francophone des 30 dernières années, dégage trois perspectives principales qui permettent d'interpréter les difficultés qu'éprouvent les élèves en mathématiques. La première perspective attribue les difficultés d'apprentissage aux caractéristiques intrinsèques à l'élève. Cette perspective s'appuie sur les fondements des

sciences cognitives. La deuxième perspective considère ces difficultés comme étant la résultante de l'interaction entre l'élève et le système didactique. Finalement, la troisième perspective se base essentiellement sur les

1 thomas Rajotte@uqar.ca 
fondements de la sociologie afin d'interpréter les difficultés des élèves en mathématiques. L'objet de cet article consiste à décrire les perspectives et à dresser un portrait des différents outils utilisés par les tenants des trois différentes perspectives explicatives des difficultés d'ens eignement et d'apprentissage en mathématiques. De plus, une brève réflexion concernant la possibilité de réaliser un arrimage entre les différentes perspectives est mise en lumière à l'intérieur de ce texte.

Mots clés: difficultés d'apprentissage; didactique des mathématiques; sociologie; approche anthropo-didactique; sciences cognitives.

\section{Resumen}

Este texto tiene por propósito destacar las contribuciones y los límites de las distintas perspectivas relacionadas con la interpretación de las dificultades de enseñanza y aprendizaje en matemáticas en los alumnos de primaria. Este artículo se sitúa más específicamente en la estela de las investigaciones que plantean una serie de cuestiones sobre la fuente principal de estas dificultades. Una mirada a los escritos científicos, sacados sobre todo delcorpus francófono de los 30 últimos años, pone de relieve tres perspectivas esenciales que permiten interpretar las dificultades que encuentran los alumnos en matemáticas. La primera perspectiva atribuye las dificultades de aprendizaje a las características inherentes al alumno. Esta perspectiva se fundamenta en las ciencias cognitivas. La secunda pers pectiva considera estas dificultades como resultante de la interacción entre el alumno y el sistema didáctico. Finalmente, la tercera perspectiva se basa esencialmente en los fundamentos de la sociología a fin de interpretar las dificultades de los alumnos en matemáticas. El objeto de este artículo pretende describir las perspectivas y mostrar el panorama de las herramientas utilizadas por los seguidores de las tres distintas perspectivas que explican las dificultades de enseñanza y aprendizaje en matemáticas. Asimismo, se presenta en este texto una breve reflexión acerca de la posibilidad de realizar un enlace entre las distintas perspectivas.

Palabras clave: dificultades de aprendizaje; didáctica de las matemáticas; sociología; enfoque antropo-didáctico; ciencias cognitivas.

\section{Resumo}

O presente texto visa salientar os aportes e limites de diferentes perspectivas relacionadas à interpretação das dificuldades de ensino e aprendizagem da matemática dos alunos do primário ${ }^{2}$. Esse artigo se situa mais precisamente no contexto das pesquisas que permitem questionar a fonte fundamental dessas dificuldades. Uma análise dos escritos científicos, publicados principalmente na literatura francófona dos últimos trinta anos, evidencia três perspectivas que permitem interpretar as dificuldades enfrentadas pelos alunos em matemática. A primeira perspectiva atribui as dificuldades de aprendizagem às características intrínsecas do aluno. Essa perspectiva é baseada nos fundamentos das ciências cognitivas. A segunda perspectiva considera essas dificuldades como sendo resultantes da interação entre aluno e sistema didático. Finalmente, a terceira pers pectiva é baseada essencialmente nos fundamentos da sociologia para interpretar as dificuldades dos alunos em matemática. Esse artigo tem por objetivo descrever as perspectivas e esboçar um retrato das diferentes ferramentas utilizadas pelos defensores das três diferentes perspectivas explicativas das dificuldades de ensino e aprendizagem em matemática. Além disso, o texto propõe uma breve reflexão sobre a possibilidade de harmonizar essas diferentes perspectivas.

Palavras-chave: dificuldades de aprendizagem; didática da matemática; sociologia; enfoque antropológico e didático; ciências cognitivas.

\section{Contexte}

Dans la foulée des travaux de la Commission des États généraux sur l'éducation, à la fin des années 1990, le ministère de l'Éducation du Québec s'est donné un défi d'envergure, soit de faire prendre à l'éducation le virage du succès (Charland, 2005; Ministère de l'Éducation du Québec [MEQ], 1997, 1999; Proulx \& Charland, 2009). Essentiellement, par cette visée, les différents acteurs du système de

\footnotetext{
2 No Québec, o ensino primário tem duração de 6 anos e é destinado às crianças de 7 a 12 anos. Ele corresponderia aos 6 primeiros anos do ensino fundamental brasileiro
} 
l'éducation devaient mettre en œuvre des actions concertées permettant de passer de l'accès du plus grand nombre au succès du plus grand nombre (Gauthier \& Saint-Jacques, 2002; MEQ, 1999).

Afin de relever le défi, des actions ciblées auprès des élèves handicapés ou en difficulté d'adaptation ou d'apprentissage (EHDAA) ont été proposées par le gouvernement de manière à soutenir la réussite de cette clientèle d'élèves reconnue comme étant la plus à risque d'échec scolaire (MEQ, 1999; Rajotte, 2014). Le besoin d'intervenir spécifiquement auprès des EHDAA est toujours d'actualité en 2017. En effet, le taux de décrochage de ce groupe d'élèves $(46,8 \%)$ est près de trois fois plus élevé que celui observé pour l'ensemble du Québec (16,2\%) (Ministère de l'Éducation, de l'Enseignement supérieur et de la Recherche, 2015). Afin de prévenir les difficultés scolaires de ces élèves, une attention particulière doit être portée à l'enseignement et à l'apprentis sa ge des mathématiques (Giroux, 2013). Cela se justifie par le fait que la société contemporaine exige des compétences mathématiques qui vont au-delà de la maîtrise d'un ensemble d'habiletés techniques (Mary, Squalli \& Schmidt, 2008; Rajotte, 2014).

Dans le but de favoriser la réussite de cette clientèle d'élèves, le ministère a publié un cadre de référence pour soutenir l'intervention des enseignants dans l'application des politiques institutionnelles en matière d'enseignement auprès des élèves en difficulté d'apprentissage (Giroux, 2013; MEQ, 2003). À cet effet, il est demandé aux enseignants d'adapter leurs interventions pédagogiques aux caractéristiques et aux besoins des EHDAA (MEQ, 1999, 2000; Ministère de l'Éducation, du Loisir et du Sport [MELS], 2007; Proulx \& Charland, 2009). Concrètement, dans le domaine des mathématiques, cette requête se traduit par la mise en œuvre d'interventions distinctes et adaptées à la situation des élèves ayant des besoins particuliers (par exemple: élèves handicapés, élèves dyspraxiques ou dyscalculiques, élèves ayant un trouble déficitaire de l'attention, élèves ayant un trouble du spectre de l'autiste) (Rajotte, Giroux \& Voyer, 2014a). Cette logique de l'adaptation découle du cadre explicatif qui relève des sciences cognitives (Giroux, 2010; Lemoyne \& Lessard, 2003; Martin \& Mary, 2010). Par contre, comme mentionné par Giroux (2007), l'application de cette recommandation est ardue puisque les enseignants disposent de peu d'appuis théoriques et de moyens didactiques permettant de réaliser cette adaptation en fonction des différents profils d'EHDAA. De plus, au courant des dernières années, les recherches sur les difficultés d'apprentissage en mathématiques ayant adopté un cadre explicatif relevant des sciences cognitives ont obtenu peu de résultats empiriques (Giroux, 2013). Conséquemment, afin d'interpréter les difficultés d'apprentissage des élèves en mathématiques, il est pertinent de considérer les fondements et les perspectives relevant d'autres disciplines qui s'intéressent à cet objet d'étude.

\section{Regard sur les différentes perspectives qui étudient les difficultés en mathématiques}

Dans le domaine des mathématiques, plusieurs écrits scientifiques révèlent trois perspectives distinctes sur la problématique des élèves présentant des difficultés d'apprentissage (Rajotte, 2015). La première perspective est essentiellement centrée sur l'identification et la description des dysfonctionnements propres à l'élève. La deuxième perspective s'intéresse plutôt au fonctionnement du système didactique et aux phénomènes qui caractérisent les relations entre la production de l'élève, la situation effective d'enseignement et la spécificité du savoir (Giroux, 2010). Finalement, la troisiè me 
perspective s'appuie sur une approche anthropo-didactique d'interprétation des difficultés d'apprentissage (Chopin, 2011; Roiné, 2012; Sarrazy, 2001). Cette perspective examine la relation enseignantélève à partir d'un double cadrage théorique (anthopologiques et didactiques) (Chevallard, 2007, 2010).

Afin de décrire la perspective adoptée par les principales disciplines qui étudient les difficultés d'apprentissage en mathématiques, Giroux (2015) a proposé un schéma permettant d'organiser ces disciplines (neuropsychologie, psychologie cognitive, psychologie développementale, didactique des mathématiques, sociologie) en fonction de leur finalité. Tel que représenté par la figure 1 , le schéma de Giroux (2015) permet de traduire les finalités de ces disciplines en situant cellesci sur un axe transversal. Sur cet axe, un déplacement vers la gauche symbolise un intérêt croissant pour l'étude du fonctionnement cognitif. Ce déplacement implique une centration sur les caractéristiques des individus. Par ailleurs, Giroux (2015) mentionne qu'un déplacement vers la droite représente un intérêt croissant pour l'étude du fonctionnement $\mathrm{du}$ savoir en situation d'enseignement ou d'apprentissage ainsi qu'une considération des facteurs culturels qui influent sur l'interprétation des difficultés des élèves. Ce mouvement engage une centration sur les phénomènes interactifs qui sont nécessaires à la transmission et à l'acquisition des savoirs.

À la lumière des propos de Giroux (2015), il est possible de dégager que les tenants de la première perspective, qui comprend particulièrement les recherches issues de la psychologie développementale, de la neuropsychologie et des sciences cognitives, se situent à la gauche de l'axe. L'attribution de cette position est justifiée

3 Sans que cela soit fixe et arrêté, il est possible de faire des liens entre les positionnements au sein du schéma de Giroux (2015) et différents paradigmes de recherche. En fait, un positionnement sur la gauche du continuum par le cadre explicatif des difficultés d'apprentissage en mathématiques, caractérisé par une centration sur les caractéristiques des individus, qui est adopté par les chercheurs œuvrant dans ces disciplines. En revanche, les tenants de la seconde perspective se localisent plus particulièrement au centre droit de l'axe dans le sens où ceux-ci effectuent une centration sur l'interaction de l'élève à l'intérieur d'un système didactique donné. Finalement, les chercheurs qui s'appuient sur les fondements de la sociologie se situent à droite de l'axe ${ }^{3}$. Ces chercheurs considèrent qu'en plus d'envisager l'influence du système didactique, il est essentiel de porter un regard sur les idéologies relevant des cultures scolaires afin d'expliquer dans quels contextes émergent les difficultés d'enseignement et d'apprentissage. Selon les tenants de cette perspective, les acteurs du milieu éducatif peuvent fabriquer des inégalités scolaires en traitant différemment les élèves caractérisés par des critères d'hétérogénéité (par exemple : élèves issus d'une famille défavorisée, élèves ayant une origine ethnique différente, élèves pratiquant une religion spécifique, etc.). pourrait être as socié au paradigme positiviste. Un positionnement au centre droit pourrait référer au constructivisme/socioconstructivisme, tandis que l'extrême droite peut être associée au paradigme socioculturel. 
Figure 1 - Schéma de Giroux (2015) concernant l'organisation des disciplines qui étudient les difficultés en mathématiques

\begin{tabular}{|c|c|c|c|c|c|c|}
\hline \multicolumn{2}{|c|}{ SCIENCES COGNITIVES } & \multirow{2}{*}{$\begin{array}{l}\text { PSYCHOLOGIE } \\
\text { DÉVELOPPE- } \\
\text { MENTALE }\end{array}$} & \multirow{2}{*}{\multicolumn{2}{|c|}{$\begin{array}{l}\text { DIDACTIQUE DES } \\
\text { MATHÉMATIQUES }\end{array}$}} & \multirow{2}{*}{\multicolumn{2}{|c|}{$\begin{array}{l}\text { ANTHROPO- } \\
\text { DIDACTIQUE }\end{array}$}} \\
\hline $\begin{array}{c}\text { NEURO- } \\
\text { PSYCHOLOGIE }\end{array}$ & $\begin{array}{l}\text { PSYCHOLOGIE } \\
\text { COGNITIVE }\end{array}$ & & & & & \\
\hline $\begin{array}{l}\text { Étude du siège } \\
\text { cérébral des } \\
\text { fonctions } \\
\text { mentales }\end{array}$ & $\begin{array}{l}\text { Étude des } \\
\text { processus } \\
\text { cognitifs/ } \\
\text { formation des } \\
\text { connaissances }\end{array}$ & $\begin{array}{l}\text { Étude du } \\
\text { développement } \\
\text { cognitif de } \\
\text { l'enfant }\end{array}$ & \multicolumn{2}{|c|}{$\begin{array}{l}\text { Étude des conditions } \\
\text { didactiques }\end{array}$} & \multicolumn{2}{|c|}{$\begin{array}{l}\text { Étude sur la } \\
\text { fabrication des } \\
\text { inégalités } \\
\text { scolaires } \\
\text { (conditions non } \\
\text { didactiques) }\end{array}$} \\
\hline \multicolumn{2}{|c|}{$\begin{array}{l}\text { CARACTÉRISTIQUES } \\
\text { DES INDIVIDUS }\end{array}$} & & \multicolumn{4}{|c|}{$\begin{array}{c}\text { TRANSMISSION/ } \\
\text { CQUISITION DE SAVOIRS }\end{array}$} \\
\hline \multicolumn{2}{|c|}{$\begin{array}{c}\text { DYSCALCULIE ET TROUBLES } \\
\text { D'APPRENTISSAGE }\end{array}$} & \multicolumn{2}{|c|}{$\begin{array}{c}\text { DIFFICULTÉ } \\
\text { D'APPRENTISSAGE }\end{array}$} & \multicolumn{3}{|c|}{$\begin{array}{c}\text { (DYS/FONCTIONNEMENTS DES } \\
\text { SYSTÈMES DIDACTIQUES) }\end{array}$} \\
\hline \multicolumn{2}{|c|}{$\begin{array}{l}\text { Mécanismes de traitement de } \\
\text { l'information (manipulation de } \\
\text { symboles) } \\
\text { - } \quad \text { Fonctionnement cognitif } \\
\text { - Déficits cognitifs }\end{array}$} & \multicolumn{2}{|c|}{$\begin{array}{l}\text { Fonctionnement } \\
\text { du savoir en situation } \\
\bullet \quad \text { Transformation } \\
\text { des connaissances }\end{array}$} & \multicolumn{2}{|c|}{$\begin{array}{l}\text { - } \\
\text { du savoir en situationnement } \\
\text { - } \quad \text { Spécificité du } \\
\text { savoir }\end{array}$} & $\begin{array}{l}\text {-Cultures } \\
\text { scolaires } \\
\text {-Idéologies } \\
\text {-Rapport au } \\
\text { savoir }\end{array}$ \\
\hline
\end{tabular}

Source: Giroux (2015).

L'évolution des législations et des politiques propres à l'adaptation scolaire tend à positionner l'orientation du ministère dans la première perspective sur les difficultés des élèves en mathématiques (Rajotte, 2014). Cette position se dégage de la Politique de l'adaptation scolaire (MEQ, 1999) qui vise à recadrer les grandes orientations de la réforme de l'éducation à l'égard des besoins particuliers et des caractéristiques propres aux EHDAA. Cette politique comprend une injonction ministérielle à l'égard des enseignants afin qu'ils adaptent leur enseignement aux caractéristiques et aux besoins des élèves (Giroux, 2013; MEQ, 1999, 2000; MELS, 2006; Proulx \& Charland, 2009).

Par ailleurs, il importe de mentionner que l'application concrète de cette politique semble occasionner d'importants défis. En effet, les modalités concernant l'adaptation des interventions pédagogiques en fonction des caractéristiques intrinsèques des élèves sont peu documentées par les écrits professionnels et scientifiques (Rajotte,
Voyer \& Giroux, 2015). Ces propos sont corroborés par Giroux (2013) ainsi que par Rajotte, Beaupré \& Beaudoin (2017) qui ajoutent que la perspective adoptée par le ministère ne se fonde pas sur une prise en compte des dimensions socioculturelle et didactique de l'enseignement. En fait, l'orientation ministérielle tend à instaurer des pratiques enseignantes visant à «combler le déficit» dont souffrirait l'élève en difficulté au détriment de la prise en compte de la spécificité relative au contenu d'enseignement, aux conditions didactiques qui favorisent les apprentissages, ainsi qu'en lien avec le contexte social qui entoure l'élève (Giroux, 2013; Rajotte et al., 2017). Selon Roiné (2014), ce constat se traduit par une forme de «cécité didactique »dans le sens où la prise en compte de la spécificité de l'enseignement et des déterminants de la situation didactique devient seconde au regard de la volonté de combler le déficit repéré chez chacun des élèves en difficulté.

Dans ce contexte, afin d'interpréter les difficultés des élèves en mathématiques, la considération des approches relevant de 
la didactique et du domaine de la sociologie paraît tout à fait justifiée. En prenant en compte les éléments positifs et négatifs de chacune des perspectives, il est pertinent d'envisager la possibilité de mettre en place un arrimage entre les différentes perspectives afin d'obtenir une meilleure interprétation des difficultés vécues par les élèves en mathématiques. Par ailleurs, avant d'envisager une telle démarche, il importe de discuter des forces et des faiblesses qui relèvent de chacune de ces perspectives. De plus, afin de décrire les différentes perspectives, il convient de présenter les principaux outils utilisés par chacune de celles-ci.

Dans les prochaines sections, l'ensemble de ces informations sera présenté sous forme de portraits succincts. Ces informations ont été recueillies par le biais d'une consultation des principales bases de données en éducation (EBSCO, SCOPUS et Proquest-Dissertations \& Theses Global). Cette démarche a permis de cibler une trentaine d'écrits scientifiques issus de trois horizons sociogéographiques principaux : les textes québécois, les textes franco-européens et, dans une moindre mesure, les textes états-uniens ${ }^{4}$.

\subsection{Première interprétative: portrait de la perspective relevant des sciences cognitives}

Comme mentionné précédemment, les tenants de la perspective associée aux sciences cognitives attribuent les difficultés d'apprentissage directement à l'élève (Rajotte, Giroux \& Voyer, 2014b). En fait, celles-ci paraissent intrinsèquement liées aux caractéristiques fonctionnelles et cognitives de l'apprenant (Lemoyne \& Lessard, 2003; Roiné, 2014). En adoptant

4 Par ailleurs, il importe de mentionner que la réflexion présentée ici ne repose pas uniquement sur cet ensemble original de textes. En effet, des textes se sont ajoutés tout au long de la démarche, tandis que plusieurs autres ont été délaissés. De ce point de vue, l'élève est perçu comme étant un sujet pour lequel les caractéristiques personnelles peuvent être mesurées par le biais d'instruments d'évaluation standardisés. Toujours selon cette perspective, le rôle de l'enseignant consiste à aider l'élève à pallier ses difficultés par le biais d'interventions de remédiation visant à modifier ses processus cognitifs (Rajotte et al., 2014b). Les principaux outils utilisés par les tenants de cette perspective ainsi que certaines critiques qui leur sont associés sont présentés ci-après.

\subsubsection{Principaux outils utilisés par les tenants de l'approche relevant des sciences cognitives}

Afin d'interpréter les difficultés d'apprentissage en mathématiques des élèves du primaire, les tenants de la perspective associée aux sciences cognitives utilisent deux principaux outils, soit: l'imagerie cérébrale ainsi que les instruments d'évaluation standardisés. À cet effet, le recours à l'imagerie cérébrale constitue un outil de prédilection pour les psychologues afin de diagnostiquer une difficulté d'apprentissage. Qu'elles soient innées ou acquises à la suite de lésions ou de traumatismes, l'utilisation de l'imagerie cérébrale permet de documenter l'activité cérébrale et de dégager si les régions du cerveau impliquées dans le traitement de l'arithmétique fonctionnent adéquatement. Selon Molko, Wilson \& Dehaene (2005), les zones cérébrales impliquées dans le traitement numérique correspondent au lobe pariétal et frontal (addition, soustraction ou multiplication) ainsi qu'au sillon intrapariétal (perception et manipulations de quantités). L'incapacité partielle d'un individu à activer ces zones cérébrales lors

cette manière, le corpus a été élargi par de nouvelles lectures, mais il a été également réduit par la mise de côté de certains textes qui ne portaient pas sur les idées qui ont été choisies afin d'être rapportées au sein de ce texte. 
de la réalisation de tâches d'arithmétique constitue un élément déterminant dans l'attribution d'une difficulté d'apprentissage pour les tenants de la perspective des sciences cognitives. À ce sujet, Sousa (2010) ainsi que Castelli, Glaser \& Butterworth (2006) ajoutent que les enfants ayant reçu un diagnostic de dyscalculie activent plus difficilement certaines zones cérébrales lors de la réalisation de tâches impliquant des estimations. Comme l'illustre la figure 2, l'étude de Kucian, Loenneker, Dietrich,
Dosch, Martin \& Von Aster (2006) relève que, pendant un travail cognitif impliquant la réalisation d'une estimation, les hémisphères droit (D) et gauche $(G)$ des enfants ayant reçu un diagnostic de dyscalculie s'activent moins que chez les enfants n'ayant pas reçu ce diagnostic. Par ailleurs, lorsque ces élèves effectuent une tâche impliquant un calcul exact, aucune différence n'est relevée au niveau de l'activation cérébrale.

Figure 2 - Représentations des zones cérébrales sollicitées lors de la réalisation de tâches impliquant des calculs exacts ou approximatifs (Kucian et al., 2006).

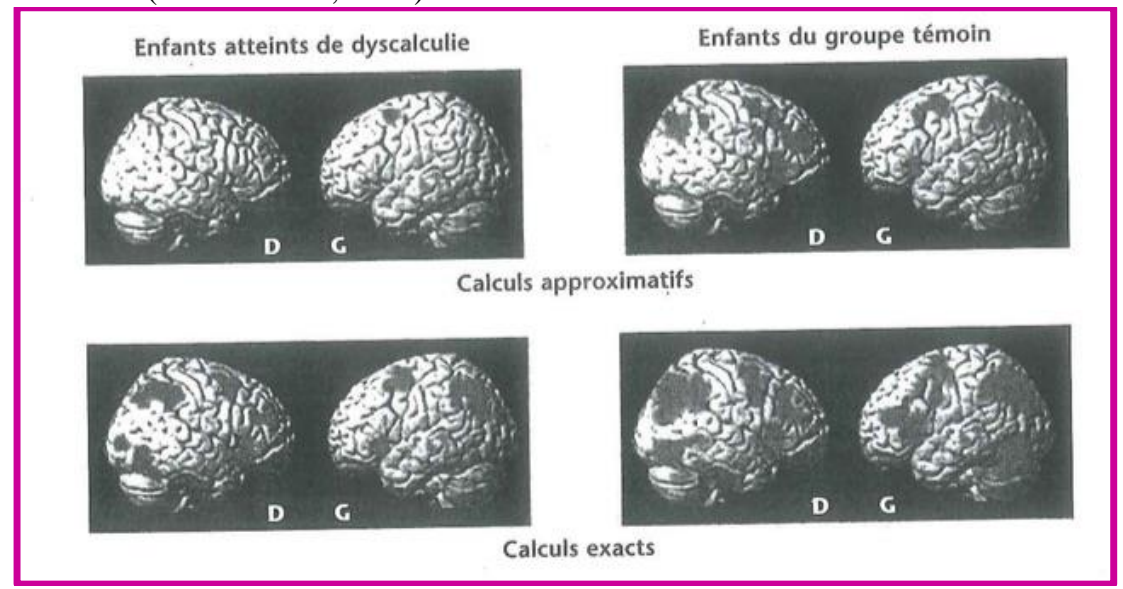

Source: Kucian et al., 2006.

De plus, les outils standardisés sont aussi fréquemment utilisés par les partisans de la première perspective. Pour les populations francophones, ces outils ont été normés sur des populations d'enfants variant en taille d'échantillons (entre 250 et 1600 enfants) et en âges (de 3 à 18 ans) (Lafay, St-Pierre \& Macoir, 2014). Ces outils visent essentiellement à situer le rendement ou la performance des enfants par le biais d'une comparaison à ses pairs qui se traduit par un rang percentile. Les six principaux outils standardisés utilisés au Québec sont présentés succinctement dans le tableau 1.

Tableau 1.

\begin{tabular}{c|c|c|c|c}
\hline & Pays/langues & Âge & $\begin{array}{c}\text { Échantillon de } \\
\text { normalis ation }\end{array}$ & $\begin{array}{c}\text { Domaines mathématiques } \\
\text { couverts }\end{array}$ \\
\hline $\begin{array}{c}\text { Key-Math- 3 } \\
\text { (Connolly, 2008) }\end{array}$ & États-Unis & $\begin{array}{c}4 \text { à } 21 \\
\text { ans }\end{array}$ & $\begin{array}{c}1638 \text { Canadiens; } \\
377 \text { Québécois }\end{array}$ & $\begin{array}{c}\text { Arithmétique } \\
- \text { Mesure } \\
\text { - Interprétation des données } \\
\text { - Résolution de problèmes }\end{array}$ \\
\hline $\begin{array}{c}\text { WIAT-II/III } \\
\text { (Wechsler, 2005) }\end{array}$ & $\begin{array}{c}\text { États-Unis/ } \\
\text { Canada }\end{array}$ & $\begin{array}{c}6 \text { à } 17 \\
\text { ans }\end{array}$ & $\begin{array}{c}\text { Pour le Canada : } \\
304 \text { Québécois }\end{array}$ & $\begin{array}{c}\text { - Opérations numériques } \\
\text { - Raisonnement } \\
\text { mathématique }\end{array}$ \\
\hline
\end{tabular}




\begin{tabular}{|c|c|c|c|c|}
\hline & & & $\begin{array}{l}356 \text { Franco- } \\
\text { Ontariens }\end{array}$ & \\
\hline $\begin{array}{c}\text { Tedi-Math } \\
\text { (Van Nieuwenhoven, Grégoire } \\
\& \text { Noël, 2001) }\end{array}$ & $\begin{array}{l}\text { Belgique } \\
\text { francophone }\end{array}$ & 5 à 8 ans & 583 & $\begin{array}{c}\text { - Comptine numérique et } \\
\text { comptage } \\
\text { - Dénombrement } \\
\text { - Calcul } \\
\text { - Numération décimale } \\
\text { - Dénombrement }\end{array}$ \\
\hline $\begin{array}{c}\text { Zareki-R } \\
\text { (Dellatolas et Von Aster, 2006) }\end{array}$ & France & $\begin{array}{c}6 \text { à } 11 \\
\text { ans et } \\
\text { demi }\end{array}$ & 249 & $\begin{array}{c}\text { - Comptine numérique et } \\
\text { comptage } \\
\text { - Dénombrement } \\
\text { - Calcul } \\
\text { - Résolution de problèmes }\end{array}$ \\
\hline $\begin{array}{c}\text { Numerical } \\
\text { (Gaillard, 2000) }\end{array}$ & Suisse & $\begin{array}{l}7 \text { à } 10 \\
\text { ans }\end{array}$ & 293 & $\begin{array}{c}\text { - Comptine numérique et } \\
\text { comptage } \\
\text { - Dénombrement } \\
\text { - Calcul } \\
\text { - Vocabulaire mathématique } \\
\text { - Dénombrement }\end{array}$ \\
\hline $\begin{array}{l}\text { Utilisation du nombre, version } \\
2 \text { (UDN 2) } \\
\text { (Meljac \& Lemmel, 1999) }\end{array}$ & France & $\begin{array}{l}4 \text { à } 11 \\
\text { ans }\end{array}$ & 420 & $\begin{array}{c}\text { - Comptine numérique et } \\
\text { comptage } \\
\text { - Dénombrement } \\
\text { - Calcul } \\
\text { - Vocabulaire mathématique }\end{array}$ \\
\hline
\end{tabular}

Source: Élaboré par l'auteur (2018).

\subsubsection{Critiques adressées au regard des outils relevant de la première perspective}

Bien que les outils utilisés par les tenants de la perspective associée aux sciences cognitives s'appuient sur des assises scientifiques impliquant des analyses statistiques (référence à la courbe de distribution normale des données, analyses inférentielles, etc.) ainsi qu'à la technologie informatique (imagerie mentale) (Tokuhama-Espinosa, 2010), certaines critiques sont adressées au regard des outils présentés. En effet, la normalisation des instruments d'évaluation a rarement été effectuée au Québec. Conséquemment, des divergences relevant des différents curriculums et des contenus à enseigner à divers degrés scolaires peuvent influer sur la performance d'un élève en comparant celui-ci à des élèves intégrant un système scolaire différent. De plus, Lafay et al.(2014) ainsi que Rajotte et al.(2017) ajoutent que ces outils ne permettent pas de considérer la spécificité relevant des tâches de mathématiques dans l'établissement du diagnostic de difficultés d'apprentissage chez l'élève. Afin de considérer la structure des tâches de mathématiques, ces auteurs suggèrent de référer aux différentes structures additives et multiplicatives proposées par Vergnaud (1982, 1983). Ces structures sont rattachées à la Théorie des champs conceptuels (Vergnaud, 1990), théorie qui sera présentée dans la prochaine section en tant qu'outil utilisé par les chercheurs en didactique.

\subsection{Deuxième perspective interprétative: portrait de la perspective relevant de la didactique}

Les travaux adoptant un cadre explicatif se rapportant à la didactique des mathématiques relèvent, pour leur part, de la deuxième perspective interprétative (Roiné, 2009). Au sein de cette perspective, les difficultés d'apprentissage sont 
interprétées comme étant la résultante de l'interaction de l'élève avec le système scolaire auquel il participe (Perrin-Glorian, 1993; Rajotte et al, 2014b). Conséquemment, l'enseignement est considéré du point de vue de la mise en place des conditions favorables à l'apprentissage par le biais d'interventions didactiques qui prennent en compte à la fois les connaissances mathématiques de l'élève et la spécificité du savoir (Martin \& Mary, 2010). Les principaux outils et les critiques associées à ceux-ci sont mis de l'avant au sein des prochaines sous-sections.

\subsubsection{Portrait des outils relevant de la didactique des mathématiques}

Les chercheurs en didactique des mathématiques adoptent des outils permettant une interprétation approfondie du contexte à l'intérieur duquel émergent les difficultés d'enseignement et d'apprentissage. Les outils utilisés consistent essentiellement à expliquer les difficultés en mathématiques portant un regard sur les modalités de fonctionnement de l'enseignement des mathématiques dans les classes du primaire. Pour délimiter le niveau à l'intérieur duquel les analyses sont effectuées, Giroux (2013) utilise les termes «méso didactique ». Ce niveau se positionne entre les études macro social, qui utilisent de vastes échantillons afin d'interpréter des données, et le niveau micro, qui se caractérise par un regard plus approfondi à un objet de recherche (par exemple, l'étude d'un individu). Afin d'interpréter les difficultés des élèves à un niveau méso didactique, les tenants de la seconde perspective interprétative des difficultés d'apprentissage réfêrent aux théories issues des travaux qui relèvent de

5 Par ailleurs, il importe de rappeler qu'il est possible de référer à un éventail beaucoup plus élargi de théories relevant du domaine de la didactique des mathématiques.

6 Selon Brousseau (1998b), le contrat didactique permet de décrire les règles implicites ou la didactique des mathématiques. Dans le cadre de ce texte, étant donné leur fréquence d'usage dans les travaux francophones, nous avons choisi de cibler deux principales théories ${ }^{5}$. Celles-ci sont présentées succinctement à l'intérieur des paragraphes suivants.

Premier outil d'interprétation des difficultés d'apprentissage - l'apport de la théorie des situations didactiques

La première théorie de référence correspond à la théorie des situations didactiques (TSD) de Brousseau (1998b). Le concept central de cette théorie correspond au contrat didactique ${ }^{6}$. Comme mentionné par Gaudreau (2005), l'idée du contrat didactique découle de l'obligation de l'enseignant de piloter des situations d'apprentissage en prenant en considération «l'histoire personnelle » de chacun de ses élèves. Dans cette histoire, le contrat didactique se traduit par le lien explicite, mais surtout implicite, en rendant chacun responsable de ce qu'il a à gérer dans la situation et dont il doit rendre compte à l'autre. De cette manière, l'enseignant manifestera ses attentes à l'élève afin qu'il les satisfasse. Puis, il proposera une situation qui exigera que l'élève mette en œuvre une connaissance visée par l'enseignant, qu'elle soit en cours d'apprentissage ou déjà connue.

Les travaux en didactique s'étant intéressés aux difficultés d'apprentissage en mathématiques ont permis de dégager que certaines difficultés vécues par les élèves pourraient, notamment, être attribuables à des effets du contrat didactique (Giroux, 2013; Martin \& Mary, 2010). Ces effets se traduisent par des phénomènes didactiques qui témoignent de la manière dont les

explicites qui régissent le partage des responsabilités, concernant la transmission ou l'acquisition du savoir, entre l'enseignant et l'élève. Ce contrat constitue donc une représentation des attentes de part et d'autre. 
contenus d'enseignement sont affectés, transformés par des intentions d'enseignement adapté aux élèves ayant des difficultés en mathématiques pour lesquels l'enseignement régulier, avec ses méthodes, a échoué (Giroux, 2007). La connaissance des différents phénomènes didactiques susceptibles de s'opérer auprès des élèves ayant des difficultés d'apprentissage en mathématiques constitue un outil pour le chercheur puisque celle-ci lui permet d'interpréter différemment (par des concepts spécifiques) la réalité du vécu scolaire de cette catégorie d'élèves. Par souci de concision, une liste non exhaustive $^{7}$ des phénomènes relevés par les écrits scientifiques est présentée ci-après:

- L'algorithmisation des objets du savoir : une règle à appliquer est enseignée au détriment de la construction de sens (Giroux, 2007);

- L'effet pharmakeia (en référence au grec classique) : implantation d'un nombre exagéré de mesures de soutien qui vient nuire au cheminement des élèves. Selon Roiné (2009), ce phénomène s'explique par le biais d'une analogie à la consommation de la pharmakeia (qui a donné «pharmacie »). Selon les cas, les circonstances et les doses employées, la pharmekeia est susceptible d'exercer une action favorable (remède) ou défavorable (poison) sur les personnes (Roiné, 2014). En effet, en consommant une quantité trop élevée de remèdes, un individu peut s'administrer un poison;

- L'effet Topaze : l'enseignant prend à sa charge une part du travail lié à une tâche en mathématiques. De cette manière, l'enseignant abaisse le niveau

7 Afin d'obtenir une description détaillée des principaux phénomènes didactiques, il est possible de consulter le glossaire rédigé par Brousseau (1998a).

8 Le temps didactique réfère à l'introduction des objets de savoir à enseigner et à leur progression de difficulté d'une tâche afin de faire en sorte qu'un élève soit en mesure de donner la réponse attendue (Brousseau \& Sarrazy, 2002);

- L'usage abusif de l'analogie : procédé qui consiste à faciliter les apprentissages en présentant à l'élève, de manière mécanique, des tâches homogènes caractérisées par une même structure. L'usage abusif de l'analogie permet à l'élève d'obtenir un rendement élevé à court terme, mais ne garantit pas une rétention des apprentissages (Brousseau \& Sarrazy, 2002).

De plus, une autre classe de phénomènes permet d'expliquer de quelle manière s'opère l'enseignement auprès des élèves en difficulté. À cet effet, Martin \& Mary (2010), par le biais d'une recension des écrits sur les phénomènes d'enseignement qui se produisent dans l'enseignement des mathématiques, réfêrent à la progression du temps didactique ${ }^{8}$ dans les classes d'adaptation scolaire ainsi qu'aux phénomènes didactiques qui en découlent. Certains phénomènes liés au temps didactique sont avancés par ces deux auteurs:

- Économie dans l'exposé du savoir : phénomène contribuant au ralentissement du temps didactique qui implique que l'enseignement de certains contenus mathématiques aux élèves en difficulté est réalisé avec moins de profondeur ou de manière plus abrégée qu'aux élèves «tout-venant » (René de Cotret \& Giroux, 2003);

- Morcellement des savoirs : segmentation des objets de savoir sans qu'il n'y ait de possibilité de construire

dans la classe. Pour assurer cette progression, l'enseignant découpe les objets de savoir à enseigner en fonction du temps d'enseignement dont il dispose et des échéances ponctuant le rythme de la vie scolaire (René de Cotret \& Giroux, 2003). 
des liens entre les savoirs et de les associer au quotidien (Cange \& Favre, 2003);

- Exclusion des élèves en difficulté lors des échanges et des débats : lors des échanges en classe, les opérations de pensée élaborées par les élèves ayant un rendement élevé sont valorisées au détriment des opérations de pensée proposées par les élèves en difficulté (Martin \& Mary, 2010).

À cela, Rajotte et al.(2014a) ajoutent qu'il importe de considérer la possibilité que le contrat didactique, ainsi que les phénomènes d'enseignement qui en découlent, soit différencié selon les différents types d'élèves qui intègrent une classe spécifique. Concrètement, cela signifie qu'il est possible qu'un pédagogue adopte des conduites différentes auprès de certaines catégories d'élèves par exemple : les élèves à risque, les élèves ayant des difficultés d'apprentissage ou les élèves identifiés comme étant inattentifs.

Deuxième outil - référence à la théorie des champs conceptuels de Vergnaud (1990)

Par ailleurs, les tenants de la seconde perspective qui souhaitent expliquer les difficultés en mathématiques des élèves du primaire peuvent aussi référer à la théorie des champs conceptuels (TCC) de Vergnaud (1990). Bien que cette théorie peut servir d'assise aux travaux des psychologues ou des didacticiens (Gaudreau, 2005), dans le présent article, cette théorie est considérée comme étant un outil des chercheurs associés à la seconde perspective interprétative des difficultés d'enseignement et d'apprentissage. Ce choix se justifie par le fait que la TCC permet de caractériser la nature et la spécificité des tâches mathématiques proposées aux élèves. Dès lors, cette théorie constitue un outil permettant de mieux comprendre les modalités relevant de l'apprentissage des mathématiques. Dans cette théorie, la notion de «champ conceptuel» vise à représenter la logique d'apprentissage, laquelle découle des liaisons nécessaires entre les notions ou les concepts mathématiques, tout en concevant que l'apprentissage est dépendant d'invariants qui sont implicites dans les procédures des sujets en situation (Rajotte et al., 2014a; Vincent, 2006). En d'autres termes, un champ conceptuel peut se définir comme un espace de problèmes ou de situations-problèmes dont le traitement implique des concepts ou des procédures de plusieurs des concepts et des procédures de plusieurs types en étroite connexion (Vergnaud, 1990).

En fonction de la classe (famille) de problèmes à laquelle il est confronté, l'élève mettra en œuvre un schème d'actions en vue d'atteindre un but. À ce sujet, Gaudreau (2005) et Vincent (2006) ajoutent que les schèmes des élèves se répartissent en deux classes de situations : dans la première classe de situations, l'élève dispose des connaissances nécessaires afin de traiter celles-ci, tandis que dans la seconde classe, l'élève ne maîtrise pas toutes les compétences nécessaires au traitement de ces situations. Par conséquent, la deuxième classe de situations sert d'assise à des réflexions, à des explorations et à des hésitations qui aboutiront ultimement à un succès ou à un échec.

Afin de clarifier la notion de «champ conceptuel», le tableau 2 présente les différents champs conceptuels associés aux structures additives. D'autres termes et concepts se rapportent à la théorie des champs conceptuels. Pour en saisir la teneur et la portée, il importe de consulter l'ouvrage de Vergnaud (1990). 
Tableau 2 - Présentation des principaux champs conceptuels relevant des structures additives .

Réunion

Dans cette catégorie de problèmes, les données sont en relation statique.

- Exemple : Josh a 4 bonbons et Balboa en a 2. Combien de bonbons ont-ils ensemble?

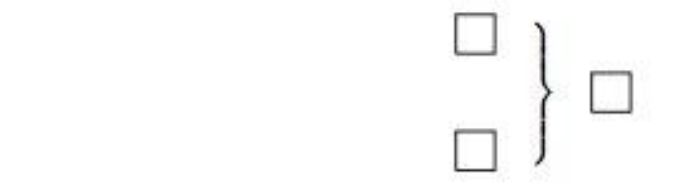

\section{Comparais on}

Dans cette classe de problèmes, les quantités de deux (ou plus) ensembles sont comparées.

- Josh a 4 bonbons. Balboa en a 2. Combien de bonbons Josh a-t-il de plus que Balboa?

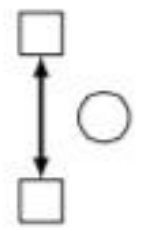

\section{Transformation d'un état}

Dans ce champ de problèmes additifs, une transformation ou un changement dans le temps agit sur un état initial pour produire un état final.

- Josh a 4 bonbons. Il en trouve 2 sur le trottoir. Combien de bonbons a-t-il maintenant?

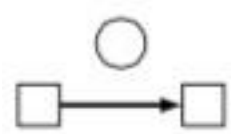

Composition de transformations

Dans cette catégorie, ni l'état initial ni l'état final ne sont connus. Le calcul porte sur une transformation qui est elle-même la combinaison.

- Josh a gagné 4 billes de plus que Balboa. Balboa en avait gagné 2. Combien de billes Josh a-t-il gagné?

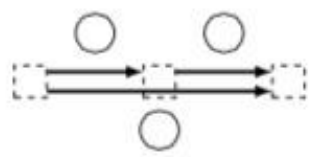

Source: Vergnaud (1990).

\subsubsection{Critiques adressées au regard des outils relevant de la deuxième perspective}

Divers travaux de recherche ont permis de documenter le rôle des phénomènes didactiques ainsi que l'influence de la structure des tâches de mathématiques sur le rendement des élèves. La considération de ces éléments peut être fort pertinente afin d'expliquer dans quels contextes peuvent émerger les difficultés d'enseignement et d'apprentissage en mathématiques. Par contre, des biais sont aussi adressés à cette seconde perspective interprétative des difficultés en mathématiques. En effet, les travaux issus de cette perspective appellent principalement la mise en œuvre d'analyses approfondies, effectuées auprès d'échantillons restreints d'individus, qui permettent difficilement de généraliser des résultats auprès de vastes échantillons d'élèves (Giroux, 2013). Confrontée à ce constat, Giroux (2013) mentionne que la problématique de l'échec scolaire est d'une telle complexité qu'elle fait appel à des outils d'analyse provenant des sciences sociales. Les outils relevant de cette discipline se rapportent à la troisième perspective interprétative des difficultés d'apprentissage. Celle-ci sera présentée dans la prochaine section. 


\subsection{Troisième}

perspective interprétative: portrait de la perspective relevant de la sociologie

La troisième perspective interprétative repose sur les thèses sociologiques explicatives de l'échec scolaire dont l'origine remonte bientôt à 35 ans (Bourdieu \& Passeron, 1985; Giroux, 2013). Les outils associés à cette perspective sont présentés dans les paragraphes suivants.

\subsubsection{Portrait des outils relevant des sciences sociales}

La perspective sociologique s'appuie sur l'approche anthropodidactique (Chevallard, 2007, 2010) afin d'expliquer les difficultés d'apprentissage en mathématiques des élèves du primaire. Cette approche se situe au croisement de deux champs théoriques : l'un didactique qui étudie les phénomènes d'enseignement en considérant le rôle central que joue la structure du savoir mathématique ainsi que les modalités concernant l'enseignement et l'apprentissage (Brousseau, 1998b; Giroux, 2013) et l'autre anthropologique qui situe son objet d'étude sur la dimension culturelle relevant des différents contextes d'enseignement qui préside aux pratiques des enseignants et des élèves, en considérant notamment l'arrière-plan cuturel qui relève du processus de socialisation d'un individu tout au long de son développement (Roiné, 2012; Sarrazy, 2002). Cet arrière-plan culturel est teinté par les «connaissances et les croyances» (Crahay, Wanlin, Issaieva et Laduron, 2010) que les enseignants ont de leurs élèves, de leur métier, de l'enseignement aux élèves en difficultés en mathématiques. Celui-ci influe, de manière inconsciente, sur l'acte d'enseigner (Roiné, 2012).

Concernant l'interprétation des difficultés d'apprentissage des élèves du primaire, cette approche considère trois dimensions (Chopin \& Sarrazy, 2010):

1) didactique, en conformité aux savoirs que le pédagogue est tenu d'enseigner selon le curriculum scolaire; 2) institutionnelle, en référence aux mœurs et coutumes qui relèvent de la culture d'appartenance des élèves et de l'enseignant; 3) pédagogique, qui consiste à mettre en œuvre une pédagogie différenciée permettant de favoriser le succès du plus grand nombre d'élèves.

En se fondant sur les théories de Bourdieu (2002), cette perspective met en relation les inégalités scolaires et les inégalités sociales (Rajotte, 2014) par une mise en lumière des mécanismes par lesquels l'institution scolaire agit comme système de reproduction des inégalités (Van Haecht, 2006). Comme mentionné précédemment, la thèse avancée par les tenants de cette perspective est que l'institution scolaire transforme le classement social des élèves en classement scolaire (Giroux, 2013) ou, autrement dit, transforme les différences de classes sociales en différences d'intelligence (Rajotte, 2014). Selon Van Haecht (2006), cette reproduction des classes s'effectuera it par le biais d'une «violence symbolique» ${ }^{9}$, dissimulant les

processus, les dominés intègrent la vision que les dominants ont du monde. Cela les amène à développer une image négative d'eux-mêmes. Par conséquent, la violence 
rapports de force entre les différentes classes sociales, qui amèneraient les classes supérieures à préserver leur statut au fil des générations.

\subsubsection{Critiques adressées à l'approche associée à la troisième perspective}

Étant donné que la perspective d'ordre sociologique a récemment émergé des travaux européens sur les difficultés d'apprentissage, peu de critiques ont été formulées à l'égard de celle-ci. Afin d'attribuer plus de valeur à la perspective associée aux sciences sociales, il pourrait être pertinent d'éprouver l'approche anthropodidactique des difficultés d'apprentissage par le biais d'études empiriques.

\section{Conclusion}

Un regard sur les outils utilisés par les différentes perspectives interprétatives, permettant d'interpréter les difficultés d'enseignement et d'apprentissage en mathématiques ainsi que les biais associés à chacune de celles-ci, amène plusieurs questionnements. Effectivement, le fait de considérer exclusivement une seule perspective interprétative, afin d'expliquer la nature des difficultés en mathématiques, pourrait engendrer une forme de «cécité interprétative» (Rajotte et al., 2014a). À ce sujet, Fisher (2009) souligne qu'il est possible que les modalités dictant l'intervention ne doivent pas être expliquées exclusivement à partir d'une seule perspective, mais plutôt à partir d'un arrimage entre les perspectives interprétatives. Ainsi, afin d'optimiser l'intervention auprès des élèves ayant des difficultés d'apprentissage en mathématiques, il est possible qu'une coopération soit nécessaire entre les différentes disciplines qui étudient ce domaine d'étude.

Bien que les différentes perspectives qui étudient les difficultés d'apprentissage adoptent des positions antagoniques, qui peuvent paraitre tout à fait inconciliables, il est possible que la meilleure façon d'interpréter les difficultés des élèves découle d'un apport complémentaire des autres perspectives. Puisque l'approche anthropo-didactique considère que les difficultés des élèves doivent être interprétées à partir d'un double ancrage théorique, didactique et sociologique, l'arrimage des fondements relevant des disciplines de recherche s'intéressant aux difficultés d'apprentissage paraît possible. De manière à opérer un éventuel arrimage entre les perspectives, il serait pertinent de réfléchir à l'élaboration d'un dispositif théorique permettant de considérer à la fois les caractéristiques individuelles de l'élève, les particularités qui relèvent du milieu d'enseignement ainsi que les variables culturelles caractérisant le contexte d'enseignement et d'apprentissage. Aussi, afin d'amener les tenants des trois perspectives à collaborer ensemble de manière à mettre en place un arrimage permettant de concilier les positions de tout et chacun, il serait nécessaire de sensibiliser les chercheurs des différentes perspectives quant aux différents champs de recherche traitant des difficultés d'enseignement et d'apprentissage (neuropsychologie, psychologie cognitive, psychologie développementale, didactique, sociologie) ainsi qu'aux outils d'interprétation qui en découlent.

De plus, en référant à la recension d'écrits menée par Martin \& 
Mary (2010), il importe de mentionner que la considération parallèle des différents phénomènes d'enseignement semble tout à fait à propos. Plus spécifiquement, un regard sur le temps didactique, ainsi que sur les phénomènes didactiques en découlant, permettrait d'adopter une vision plus globale quant aux différents facteurs susceptibles d'interférer dans l'interprétation des difficultés des élèves en mathématiques. Bien que la considération des phénomènes d'enseignement relève principalement de la perspective interprétative associée à la didactique, il est aisément concevable d'envisager des liens avec d'autres perspectives. En effet, il serait possible de référer aux phénomènes d'enseignement susceptibles de se produire auprès des élèves ayant des caractéristiques spécifiques (cela implique de considérer que le contrat didactique peut être modulé selon le type d'élève), ainsi qu'en fonction de «l'arrière-plan culturel », découlant du processus de socialisation d'un individu, qui influe sur ses actes et influence ses choix pédagogiques. Avant d'arriver à cette fin, des travaux subséquents et de nombreuses activités de diffusion devront être mis en oeuvre.

\section{Références bibliographiques}

Bourdieu, P. (2002). Intervention, 19612001, Science sociale et action politique. Marseille: Agone.

Bourdieu, P. \& Passeron, J.C. (1970). La reproduction. Éléments pour une théorie $d u$ système d'enseignement. Paris: Les Éditions de Minuit.

Bourdieu, P. \& Passeron, J.C. (1985). Les héritiers: les étudiants et la culture. Paris: Les Éditions de Minuit.
Brousseau, G. (1998a). Glossaire de quelques concepts de la théorie des situations didactiques en mathématiques (1998). Récupéré de: <guy-brousseau.com/wpcontent/uploads/2010/09/Glossaire _V5.pdf>.

Brousseau, G. (1998b). Théorie des situations didactiques. Grenoble: La pensée sauvage.

Brousseau, G. \& Sarrazy, B. (2002). Glossary of Terms Used in Didactique. Université Bordeaux 2, Bordeaux.

Cange, C. \& Favre, J.-M. (2003). L'enseignement des mathématiques dans l'enseignement spécialisé est-il pavé de bonnes analyses d'erreurs? Éducation et francophonie, 31(2), 199-217.Castelli, F.

Glaser, D.E. \& Butterworth, B. (2006). Discrete and analogue quantity proceeding in the parietal lobe: A functional MRI study. Proceedings of the National Academy of Sciences, 103(12), 4693-4698.

Charland, J.P. (2005). Histoire de l'éducation au Québec: de l'ombre du clocher à l'économie du savoir. Montréal: Éditions du Renouveau pédagogique.

Chevallard, Y. (2007). Passé et présent de la théorie anthropologique du didactique. Actes du congrès Sociedad, Escuela y Matemáticas. Aportaciones de la teoría antropológica de lo didáctico. (p.705-746). Baeza, Espagne.

Chevallard, Y. (2010). «La didactique, dites-vous?», Éducation et didactique, 4(1), 139-148. 
Chopin, M.P. (2011). Le temps de l'enseignement. L'avancée $d u$ savoir et la gestion des hétérogénéités dans la classe. Rennes: Presses Universiaires de Rennes, coll. «Paideia».

Chopin, M.P. \& Sarrazy, B. (2010). Contribution à l'évaluation de l'individualisation de l'enseignement: une étude anthropo-didactique des fonctions des interactions phatiques dans l'enseignement de l'arithmétique en cycle 3. Actes du congrès de l'actualité de la recherche en éducation et en formation (AREF), Genève.

Connolly, A.J. (2008). KeyMath ${ }^{T M}, 3 e$ édition canadienne. Toronto: Pearson.

Crahay, M., Wanlin, P., Issaeiva, É. \& Laduron, I. (2010). Fonctions, structuration et évolution des croyances (et connaissances) des enseignants. Revue française de pédagogie, 172, 85-129.

Dellatolas, G. \& Von Aster, M. (2006). Zareki-R: batterie pour l'évaluation du traitement des nombres et du calcul chez l'enfant. Paris: Les Éditions du Centre de psychologie appliquée.

Fisher, J.P. (2009). Six questions ou propositions pour cerner la notion de difficulté développementale. Approche neuropsychologique des apprentissages chez l'enfant, 21(2), 117-133.

Gaillard, F. (2000). Numerical, test neurocognitif pour l'apprentissage du nombre et du calcul. Actualités psychologiques (édition spéciale). Institut de psychologie. Université de Lausanne, Lausanne.
Gaudreau, A. (2005). Échecs en math? Dépistage et intervention auprès des élèves à risque au préscolaire et au premier cycle. Montréal: Éditions Hurtubise HMH Ltée.

Gauthier, C. \& Saint-Jacques, D. (2002). La réforme des programmes scolaires au Québec. Québec: Presses de l'Université Laval.

Giroux, J. (2007). Adapter l'enseignement en classe d'adaptation scolaire (LA TSD à la rescousse des difficultés d'enseignement aux élèves en difficultés d'apprentissage). Actes du symposium de Bordeaux 2, Entre didactique et politique: Actualités de la théorie des situations didactiques à propos de quelques questions vives sur l'enseignement des mathématiques à l'école élémentaire, Bordeaux.

Giroux, J. (2010). Pour une différenciation de la dyscalculie et des difficultés d'apprentissage en mathématiques. Actes de colloque $\mathrm{du}$ Groupe de didactique des mathématiques, Moncton.

Giroux, J. (2013). Étude des rapports enseignement/apprentissage des mathématiques dans le contexte de l'adaptation scolaire: problématique et repères didactiques. Éducation et Didactique, 7(1), 59-86.

Giroux, J. (2015). Difficultés des élèves en mathématiques: apports de la didactique. Conférence à la Haute école pédagogique du Canton de Vaud. Unité d'enseignement et de recherche Didactique des mathématiques et des sciences de la nature, Suisse, $1^{\mathrm{er}}$ octobre. 
Kucian, K.T., Loenneker, T., Dietrich, M., Dosch, E., Martin, E. \& von Aster, M. (2006). Impaired neural networks for approximate calculation in dyscalculic children: A functional MRI study. Behavioral and Brain Functions, 2, 31-48.

Lafay, A., St-Pierre, M.-C. \& Macoir, J. (2014). L'évaluation des habiletés mathématiques de l'enfant: inventaire critique des outils disponibles. Glossa, 116, 33-58.

Lemoyne, G. \& Lessard, G. (2003). Les rencontres singulières entre les élèves présentant des difficultés d'apprentissage en mathématiques et leurs enseignants. Éducation et francophonie, 21(2), 13-44.

Martin, V. \& Mary, C. (2010). Particularités de l'enseignement des mathématiques à des élèves en classes régulières ou spéciales. Actes de colloque du Groupe de didactique des mathématiques, Moncton.

Mary, C., Squalli, H.\& Schmidt, S. (2008). Mathématiques et élèves en difficulté grave d'apprentissage: Contexte favorable à l'intégration et au raisonnement mathématique. In J.M. Bisaillon et N. Rousseau (dir.). Les jeunes en difficulté: Contextes d'intervention favorables. Québec: Presses de l'Université du Québec.

Meljac, C. \& Lemmel, G. (1999). UDN2: Construction et Utilisation $d u$ nombre. Paris: Les Éditions du Centre de psychologie appliquée.

Martin, V. \& Mary, C. (2010). Particularités de l'enseignement des mathématiques à des élèves en difficulté en classes régulières ou spéciales. Actes du colloque du Groupe de didactique des mathématiques, Université de Moncton, Moncton.

Ministère de l'Éducation du Québec (1997). Réaffirmer l'école. Prendre le virage du succès. Rapport $d u$ groupe de travail sur la réforme du curriculum. Gouvernement du Québec, Québec.

Ministère de l'Éducation du Québec (1999). Une école adaptée à tous ses élèves: Politique de l'adaptation scolaire. Gouvernement du Québec, Québec.

Ministère de l'Éducation du Québec (2000). Élèves handicapés ou élèves en difficulté d'adaptation ou d'apprentissage (EHDAA): définitions. Direction de l'adaptation scolaire et des services complémentaires, Québec.

Ministère de l'Éducation du Québec (MEQ) (2003). Les difficultés d'apprentissage à l'école: Cadre de référence pour guider l'intervention. Gouvernement du Québec, Québec.

Ministère de l'Éducation, de l'Enseignement supérieur et de la Recherche (2015). Bulletin statistique de l'éducation, 43. Gouvernement du Québec, Québec.

Ministère de l'Éducation, du Loisir et du Sport (2006). L'évaluation des apprentissages au secondaire. Cadre de référence. Gouvernement du Québec, Québec.

Ministère de l'Éducation, du Loisir et du Sport (2007). L'organisation des services éducatifs aux élèves à risque et aux élèves handicapés ou en difficulté d'adaptation ou 
d'apprentissage

(EHDAA).

Gouvernement du Québec, Québec.

Molko, N., Wilson, A. \& Dehaene, S. (2005). La dyscalculie développementale, un trouble primaire de la perception des nombres. Revue française de pédagogie, 152(1), 41-47.

Perrin-Glorian, M.-J. (1993). Questions didactiques soulevées à partir de l'enseignement des mathématiques dans des classes «faibles». Recherche en didactique des mathématiques, 13(1/2), 5-18.

Proulx, J.P. \& Charland, J.P. (2009). Le système éducatif du Québec: De la maternelle à l'université. Montréal: Chenelière Éducation.

Rajotte, T. (2014). La résolution de problèmes de proportionnalité chez les élèves de sixième année $d u$ primaire avec ou sans TDA/H identifié. Thèse de doctorat en éducation. Université du Québec à Rimouski, Rimouski.

Rajotte, T. (2015). The Interpretation of Mathematical Difficulties in Primary School: Which Perspective to Choose. Journal of Literature and Art Studies, 5(10), 901-910.

Rajotte, T., Beaupré, S. et Beaudoin, D. (2017). Testing The AnthropoDidactic Approach in Order to Interpret the Learning Difficulties in Mathematics for Elementary Students. Actes du colloque international de la Clute Institute. Stockholm, Suède. (p.124-1 à 1249).

Rajotte, T., Giroux, J. et Voyer, D. (2014a). Les difficultés d'apprentissage en mathématiques au primaire: ce qu'il faut considérer pour être en mesure de bien interpréter. Saarbrücken: Presses académiques francophones.

Rajotte, T., Giroux, J. \& Voyer, D. (2014b). Les difficultés des élèves du primaire en mathématiques, quelle perspective d'interprétation privilégier? Revue des sciences de l'éducation de McGill, 49(1), 6787.

Rajotte, T.,Voyer, D. \& Giroux, J. (2015). Les difficultés en résolution de problèmes mathématiques des élèves ayant un TDA/H: quelle perspective adopter afin d'intervenir adéquatement? Revue canadienne des jeunes chercheures et chercheurs en éducation, 6(1), 122.

René de Cotret, S. \& Giroux, J. (2003). Le temps didactique dans trois classes de secondaire I (doubleurs, ordinaires, forts). Éducation et francophonie, 31(2), 155-175.

Roiné, C. (2009). Cécité didactique et discours noosphériens dans les pratiques d'enseignement en S.E.G.P.A: une contribution à la question des inégalités. Thèse inédite. Université Victor Segalen Bordeaux 2, Bordeaux.

Roiné, C. (2012). Analyse anthropo didactique de l'aide mathématique aux «élèves en difficulté»: l'effet Pharmakéia. Carrefours de l'éducation, 1(33), 131-147.

Roiné, C. (2014). Les paradoxes de l'aide aux «élèves en difficulté» dans l'enseignement des mathématiques. In $\mathrm{C}$. Mary, $\mathrm{H}$. Squalli, L. Theis et L. DeBlois (dir.). Recherches sur les difficultés d'enseignement et d'apprentissage des mathématiques: Regard 
didactique (p.45-62). Québec: Presses de l'Université du Québec.

Sarrazy, B. (2001). Les interactions maître-élèves dans l'enseignement des mathématiques. Contribution à une approche anthropo-didactique des phénomènes d'enseignement. Revue française de pédagogie, 136(1), 117-132.

Sarrazy, B. (2002). Approche anthropodidactique des phénomènes d'enseignement des mathématiques: Contribution à l'étude des inégalités scolaires à l'école élémentaire. Note de synthèse pour l'habilitation à diriger des recherches, Didactique et anthropologie des enseignements des sciences et des techniques (DAEST), Bordeaux.

Sousa, D.A. (2010). Un cerveau pour apprendre les mathématiques: Mieux comprendre le fonctionnement du cerveau pour enseigner les mathématiques plus efficacement. Montréal: Chenelière Éducation.

Tokuhama-Espinosa, T. (2010). Mind, Brain and Education Science: A Comprehensive Guide to the New Brain-Based Teaching, New York, NY: W.W. Norton \& Company.

Van Haecht, A. (2006). L'école à l'épreuve de la sociologie: La sociologie de l'éducation et ses évolutions. Bruxelles: Éditions De Boeck Université.

Van Nieuwenhoven, C., Grégoire, J. \& Noël, M.P. (2001). Tedi-Math. Test diagnostique des compétences de base en mathématiques. Paris: Les Éditions du Centre de psychologie appliquée.
Vergnaud, G. (1982). A classification of cognitive tasks and operations of thought involved in addition and subtraction problems. In T.P. Carpenter, J.M. Moser et T.A Romberg (dir.). Addition and subtraction: a cognitive perspective (p.39-59). Hilldale, NJ: National Council of Teachers of Mathematics.

Vergnaud, G. (1983). Multiplicative structures. In R. Lesh et M. Landau. (Dir.). Acquisition of mathematics concepts and processes (p.127174). New York: Academic Press.

Vergnaud, G. (1990). La théorie des champs conceptuels. Recherches en didactique des Mathématiques, 10(2/3), 133-170.

Vincent, S. (2006). Allez et multipliez. Montréal: Éditions Bande didactique.

Wechsler, D. (2005). Test de rendement individuel de Wechler (2 éd.), version pour francophone. Toronto: Pearson. 